\title{
Minimal Dominating Functions of Corona Product Graph of a Path with a Cycle
}

\author{
M. S. Vimala Devi \\ Sr. Asst, TTD \\ Tirupati, Andhra Pradesh, \\ India
}

\author{
B. Maheswari \\ Professor \\ Sri Padmavathi Mahila Viswa Vidyalayam, Tiruapti, \\ Andhra Pradesh, India
}

\begin{abstract}
Graph Theory has been realized as one of the most useful branches of Mathematics or recent origin, finding widest applications in all most all branches of Sciences, Engineering and Computer Science. An introduction and an extensive overview on domination in graphs and related topics are given by Haynes et al. [1, 2].

Recently, dominating functions in domination theory have received much attention. They give rise to important classes of graphs and deep structural problems. In this paper it is a discussion on some results on minimal dominating functions of corona product graph of a Path with a Cycle.
\end{abstract}

\section{Keywords}

Corona Product, Path, Cycle, Dominating Function

\section{INTRODUCTION}

The Domination theory is an important branch of Graph Theory and the concept of domination number of a graph is first introduced by Berge [9] in his book on graph theory. The concept of Total dominating sets are introduced by C.J.Cockayane, and Hedetniemi,S.T[4] and the concept of dominating functions introduced by Hedetniemi et al.[5].

Frutch and Harary [7] introduced a new product on two graphs $G_{1}$ and $G_{2}$, called corona product denoted by $G_{1}$ (2) $G_{2}$. The object is to construct a new and simple operation on two graphs $G_{1}$ and $G_{2}$ called their corona, with the property that the group of the new graph is in general isomorphic with the wreath product of the groups of $G_{1}$ and $G_{2}$.

Here some basic properties of corona product graph of a Path with a Cycle and some results on minimal dominating functions are presented.

\section{CORONA PRODUCT OF $P_{\boldsymbol{n}} \odot \boldsymbol{C}_{\boldsymbol{m}}$}

The corona product of a Path $P_{n}$ with a Cycle $C_{m}$, is a graph obtained by taking one copy of a n-vertex graph $P_{n}$ and n copies of $C_{m}$ and then joining the $\mathrm{i}^{\text {th }}$ vertex of $P_{n}$ to all vertices of $i^{\text {th }}$ copy of $C_{m}$ and it is denoted by $\boldsymbol{P}_{\boldsymbol{n}} \odot \boldsymbol{C}_{\boldsymbol{m}}$. Now we present some of the properties of corona product graph $\boldsymbol{P}_{\boldsymbol{n}} \odot \boldsymbol{C}_{\boldsymbol{m}}$ without proofs.

Theorem 2.1: The graph $G=P_{n} \odot C_{m}$ is a connected graph.

Theorem 2.2: The degree of a vertex $v$ in $G=P_{n} \odot C_{m}$ is given by

$$
d\left(v_{i}\right)=\left\{\begin{aligned}
m+2, & \text { if } v_{i} \in P_{n} \text { and } 2 \leq i \leq(n-1), \\
m+1, & \text { if } v_{i} \in P_{n} \text { and } i=1 \text { or } n, \\
3, & \text { if } v_{i} \in C_{m} .
\end{aligned}\right.
$$

Theorem 2.3: The number of vertices and edges in $G=$ $P_{n} \odot C_{m}$ is given respectively by

1. $|V(G)|=n(m+1)$,

2. $|E(G)|=(2 m n+n-1)$.

Theorem 2.4: The graph $G=P_{n} \odot C_{m}$ is non hamiltonian.

Theorem 2.5: The graph $G=P_{n} \odot C_{m}$ is not eulerian.

Theorem 2.6: The graph $G=P_{n} \odot C_{m}$ is not bipartite.

\section{DOMINATING SETS DOMINATING FUNCTIONS}

In this section we study dominating sets, dominating functions of the graph $G=P_{n} \odot C_{m}$ and we present some results related to minimal dominating functions of this graph.

Theorem 3.1: The domination number of $G=P_{n} \odot C_{m}$ is $n$.

Proof: Let $D$ denote a dominating set of $G$.

Case 1: Suppose $D$ contains the vertices of $P_{n}$ in $G$.

By the definition of $G$, the $i^{\text {th }}$ vertex in $P_{n}$ is adjacent to all vertices of $i^{t h}$ copy of $C_{m}$. That is the vertices in $P_{n}$ dominate the vertices in all copies of $C_{m}$ respectively. Therefore the vertices of $D$ dominate all vertices of $G$. Thus $D$ becomes a DS of $G$. This set is also minimal, because, if we delete one vertex say $v_{i}$ from $D$, then the vertices in the $i^{\text {th }}$ copy of $C_{m}$ are not dominated by any vertex in $D$. Hence $\gamma(G)=n$.

Case2: Suppose $D$ contains any one vertex of $C_{m}$ in each copy of $G$.

That is $|D|=n$. Obviously every vertex in $C_{m}$ dominates every other vertex in $C_{m}$ and also a single vertex of $P_{n}$ to which it is associated.

Therefore the vertices in $D$ dominate all vertices of $G$. Further this set is also minimal. Therefore $\gamma(G)=n$.

Theorem 3.2: Let $D$ be a MDS of $G=P_{n} \odot C_{m}$. Then a function $f: V \rightarrow[0,1]$ defined by

$$
f(v)= \begin{cases}1, & \text { if } \mathrm{v} \in D \\ 0, & \text { otherwise. }\end{cases}
$$

becomes a MDF of $G=P_{n} \odot C_{m}$.

Proof: we have seen in Theorem 3.1, that the DS of $G$ contains all the vertices of $P_{n}$ and this set is also minimum. Also the set of vertices whose degree is $m$ in each copy of $C_{m}$ 
form a minimal DS of $G$. Let $D$ be a MDS of $G$. For definiteness let $D$ contain the vertices of $P_{n}$ in $G$.

In $P_{n}$, there are two end vertices of degree $\mathrm{m}+1$ and there are n-2 intermediate vertices of degree $m+2$ respectively in $G$. In $C_{m}$, there are $m$ vertices of degree 3 respectively in $G$.

The summation value taken over $N[v]$ of $v \in V$ is as follows:

Case 1: Let $v \in P_{n}$ be such that $d(v)=m+2$ in $G$.

Then $N[v]$ contains $m$ vertices of $C_{m}$ and three vertices of $P_{n}$ in $G$.

So $\sum_{u \in N[v]} f(u)=1+1+1+\underbrace{0+\ldots \ldots .+0}_{m \text {-times }}=3$.

Case 2: Let $v \in P_{n}$ be such that $d(v)=m+1$ in $G$.

Then $N[v]$ contains $m$ vertices of $C_{m}$ and two vertices of $P_{n}$ in $G$.

So $\sum_{u \in N[v]} f(u)=1+1+\underbrace{0+\ldots \ldots+0}_{m \text {-times }}=2$.

Case 3: Let $v \in C_{m}$ be such that $d(v)=3$ in $G$.

Then $N[v]$ contains 3 vertices of $C_{m}$ and one vertex of $P_{n}$ in G.

So $\sum_{u \in N[v]} f(u)=1+0+0+0=1$.

Therefore for all possibilities, we get $\sum_{u \in N[v]} f(u) \geq 1$, $\forall \mathrm{v} \in \mathrm{V}$.

This implies that $f$ is a DF.

Now we check for the minimality of $f$.

Define $g: V \rightarrow[0,1]$ by

$g(v)$

$= \begin{cases}\mathrm{r}, & \text { if } \mathrm{v}=\mathrm{v}_{\mathrm{k}} \in \mathrm{D} \text { with } \mathrm{d}\left(\mathrm{v}_{\mathrm{k}}\right)=\mathrm{m}+1, \\ 1, & \text { if } \mathrm{v} \in \mathrm{D}-\left\{\mathrm{v}_{\mathrm{k}}\right\}, \\ 0, & \text { otherwise. }\end{cases}$

where $0<r<1$

Since strict inequality holds at the vertex $v_{k} \in D$, it follows that $g<f$.

Now the following cases arise.

Case (i): Let $v \in P_{n}$ be such that $d(v)=m+2$ in $G$.

Sub case 1:Let $v_{k} \in N[v]$.

$\sum_{u \in N[v]}^{\text {Then }} g(u)=r+1+1+\underbrace{0+\ldots \ldots . .+0}_{m \text {-times }}=r+2>1$.

Sub case 2:Let $v_{k} \notin N[v]$.
Then $\sum_{u \in N[v]} g(u)=1+1+1+\underbrace{0+\ldots \ldots+0}_{m \text {-times }}=3$.

Case (ii): Let $v \in P_{n}$ be such that $d(v)=m+1$ in $G$.

Sub case 1: Let $v_{k} \in N[v]$.

Then $\sum_{u \in N[v]} g(u)=r+1+\underbrace{0+\ldots \ldots .+0}_{m \text {-times }}=r+1>1$.

Sub case 2: Let $v_{k} \notin N[v]$.

Then $\sum_{u \in N[v]} g(u)=1+1+\underbrace{0+\ldots \ldots+0}_{m \text {-times }}=2$.

Case (iii): Let $v \in C_{m}$ be such that $d(v)=3$ in $G$.

Sub case 1: Let $v_{k} \in N[v]$.

Then $\sum_{u \in N[v]} g(u)=r+0+0+0=r<1$.

Sub case 2: Let $v_{k} \notin N[v]$.

Then $\sum_{u \in N[v]} g(u)=1+0+0+0=1$

This implies that $\sum_{u \in N[v]} g(u)<1$, for some $v \in V$.

So $g$ is not a DF.

Since $g$ is taken arbitrarily, it follows that there exists no $g<f$ such that $g$ is a DF.

Thus $f$ is a MDF.

Theorem 3.3: A function $f: V \rightarrow[0,1]$ defined by $f(v)=$ $\frac{1}{q}, \forall v \in V$ is a DF of $G=P_{n} \odot C_{m}$ if $q \leq 4$. It is a MDF if $q=4$.

Proof: Let $f$ be a function defined as in the hypothesis.

We know that in $P_{n}$, there are two end vertices of degree $m+1$ and there are $n-2$ intermediate vertices of degree $m+2$ respectively in $G$. In $C_{m}$, there are $m$ vertices of degree 3 respectively in $G$.

Case I: Suppose $0<q<4$.

The summation value taken over $N[v]$ of $v \in V$ is as follows:

Case 1: Let $v \in P_{n}$ be such that $d(v)=m+2$ in $G$.

Then $N[v]$ contains $m$ vertices of $C_{m}$ and three vertices of $P_{n}$ in $G$.

So $\sum_{u \in N[v]} f(u)=\underbrace{\frac{1}{q}+\frac{1}{q}+\ldots \ldots+\frac{1}{q}}_{(m+3) \text {-times }}=\frac{m+3}{q}$.

Since $q<4$, it follows that $\frac{m+3}{q}>1$.

Case 2: Let $v \in P_{n}$ be such that $d(v)=m+1$ in $G$. 
Then $N[v]$ contains $m$ vertices of $C_{m}$ and two vertices of $P_{n}$ in $G$.

So $\sum_{u \in N[v]} f(u)=\underbrace{\frac{1}{q}+\frac{1}{q}+\ldots \ldots+\frac{1}{q}}_{(m+2) \text {-times }}=\frac{m+2}{q}$.

Since $q<4$, it follows that $\frac{m+2}{q}>1$.

Case 3: Let $v \in C_{m}$ be such that $d(v)=3$ in $G$.

Then $N[v]$ contains 3 vertices of $C_{m}$ and one vertex of $P_{n}$ in G.

So $\sum_{u \in N[v]} f(u)=\frac{1}{q}+\frac{1}{q}+\frac{1}{q}+\frac{1}{q}=\frac{4}{q}$.

Since $q<4$, it follows that $\frac{4}{q}>1$.

Therefore for all possibilities, we get $\sum_{u \in N[v]} f(u)>1$,

$$
\forall \mathrm{v} \in \mathrm{V} .
$$

This implies that $f$ is a DF.

Now we check for the minimality of $f$.

Define $g: V \rightarrow[0,1]$ by

$g(v)=\left\{\begin{array}{l}\mathrm{r}, \text { if } \mathrm{v}=\mathrm{v}_{\mathrm{k}} \in \mathrm{D} \text { with } \mathrm{d}\left(\mathrm{v}_{\mathrm{k}}\right)=\mathrm{m}+1, \\ \frac{1}{\mathrm{q}}, \text { otherwise. }\end{array}\right.$

where $0<r<\frac{1}{q}$.

Since strict inequality holds at a vertex $v_{k}$ of $V$, it follows that $g<f$.

Case (i): Let $v \in P_{n}$ be such that $d(v)=m+2$ in $G$.

Sub case 1:Let $v_{k} \in N[v]$.

$$
\text { Then } \begin{aligned}
\sum_{u \in N[v]} g(u)= & r+\underbrace{\frac{1}{q}+\frac{1}{q}+\ldots \ldots+\frac{1}{q}}_{(m+2) \text {-times }} \\
& <\frac{1}{q}+\frac{m+2}{q}=\frac{m+3}{q} .
\end{aligned}
$$

Since $q<4$, it follows that $\frac{m+3}{q}>1$.

Sub case 2:Let $v_{k} \notin N[v]$.
Then $\sum_{u \in N[v]} g(u)=\underbrace{\frac{1}{q}+\frac{1}{q}+\ldots \ldots .+\frac{1}{q}}_{(m+3) \text {-times }}=\frac{m+3}{q}>1$,

since $q<4$.

Case (ii): Let $v \in P_{n}$ be such that $d(v)=m+1$ in $G$.

Sub case 1:Let $v_{k} \in N[v]$.

Then $\sum_{u \in N[v]} g(u)=r+\underbrace{\frac{1}{q}+\frac{1}{q}+\ldots \ldots+\frac{1}{q}}_{(m+1) \text {-times }}$

since $\mathrm{q}<4$.

$$
<\frac{1}{q}+\frac{m+1}{q}=\frac{m+2}{q}>1
$$

Sub case 2:Let $v_{k} \notin N[v]$.

Then $\sum_{u \in N[v]} g(u)=\underbrace{\frac{1}{q}+\frac{1}{q}+\ldots \ldots .+\frac{1}{q}}_{(m+2) \text {-times }}=\frac{m+2}{q}>1$, since $\mathrm{q}<4$.

Case (iii): Let $v \in C_{m}$ be such that $d(v)=3$ in $G$.

Sub case 1:Let $v_{k} \in N[v]$.

Then $\sum_{u \in N[v]} g(u)=r+\frac{1}{q}+\frac{1}{q}+\frac{1}{q}$

$<\frac{1}{q}+\frac{3}{q}=\frac{4}{q}>1$, since $\mathrm{q}<4$.

Sub case 2: Let $v_{k} \notin N[v]$.

Then $\sum_{u \in N[v]} g(u)=\frac{1}{q}+\frac{1}{q}+\frac{1}{q}+\frac{1}{q}=\frac{4}{q}>1$.

Hence it follows that $\sum_{u \in N[v]} g(u) \geq 1, \forall \mathrm{v} \in \mathrm{V}$.

Thus $g$ is a DF.

This implies that $f$ is not a MDF.

Case II: Suppose $q=4$.

The summation value taken over $N[v]$ of $v \in V$ is as follows:

Case 1: Let $v \in P_{n}$ be such that $d(v)=m+2$ in $G$.

Then $N[v]$ contains $m$ vertices of $C_{m}$ and three vertices of $P_{n}$ in $G$. 
So

$\sum_{u \in N[v]} f(u)=\underbrace{\frac{1}{q}+\frac{1}{q}+\ldots \ldots+\frac{1}{q}}_{(m+3)-\text { times }}=\frac{m+3}{q}=\frac{m+3}{4}>1$, since $\mathrm{q}=4$.

Case 2: Let $v \in P_{n}$ be such that $d(v)=m+1$ in $G$.

Then $N[v]$ contains $m$ vertices of $C_{m}$ and two vertices of $P_{n}$ in $G$.

So

$\sum_{u \in N[v]} f(u)=\underbrace{\frac{1}{q}+\frac{1}{q}+\ldots \ldots .+\frac{1}{q}}_{(m+2) \text {-times }}=\frac{m+2}{q}=\frac{m+2}{4}>1$, since $\mathrm{q}=4$.

Case 3: Let $v \in C_{m}$ be such that $d(v)=3$ in $G$.

Then $N[v]$ contains 3 vertices of $C_{m}$ and one vertex of $P_{n}$ in $G$.

So

$\sum_{u \in N[v]} f(u)=\frac{1}{q}+\frac{1}{q}+\frac{1}{q}+\frac{1}{q}=\frac{4}{q}=\frac{4}{4}=1$, since $\mathrm{q}=4$.

Therefore for all possibilities, we get $\sum_{u \in N[v]} f(u) \geq 1$, $\forall \mathrm{V} \in \mathrm{V}$.

This implies that $f$ is a DF.

Now we check for the minimality of $f$.

Define $g: V \rightarrow[0,1]$ by

$g(v)=\left\{\begin{array}{l}\mathrm{r}, \text { if } \mathrm{v}=\mathrm{v}_{\mathrm{k}} \in \mathrm{D} \text { with } \mathrm{d}\left(\mathrm{v}_{\mathrm{k}}\right)=\mathrm{m}+1, \\ \frac{1}{\mathrm{q}}, \text { otherwise. }\end{array}\right.$

where $0<r<\frac{1}{q}$.

Since strict inequality holds at a vertex $v_{k}$ of $V$, it follows that $g<f$.

Case (i): Let $v \in P_{n}$ be such that $d(v)=m+2$ in G.

Sub case 1:Let $v_{k} \in N[v]$.

$$
\begin{aligned}
& \text { Then } \sum_{u \in N[v]} g(u)=r+\underbrace{\frac{1}{q}+\frac{1}{q}+\ldots \ldots+\frac{1}{q}}_{(m+2) \text {-times }} \\
& <\frac{1}{q}+\frac{m+2}{q}=\frac{m+2}{q}=\frac{m+2}{4}>1 .
\end{aligned}
$$

Sub case 2:Let $v_{k} \notin N[v]$.

$$
\text { Then } \begin{aligned}
\sum_{u \in N[v]} g(u)= & \underbrace{\frac{1}{q}+\frac{1}{q}+\ldots \ldots+\frac{1}{q}}_{(m+3) \text {-times }} \\
& =\frac{m+3}{q}=\frac{m+3}{4}>1 .
\end{aligned}
$$

Case (ii): Let $v \in P_{n}$ be such that $d(v)=m+1$ in $G$.

Sub case 1:Let $v_{k} \in N[v]$.

Then $\sum_{u \in N[v]} g(u)=r+\underbrace{\frac{1}{q}+\frac{1}{q}+\ldots \ldots+\frac{1}{q}}_{(m+1) \text {-times }}$

$$
<\frac{1}{q}+\frac{m+1}{q}=\frac{m+2}{q}=\frac{m+2}{4}>1 \text {. }
$$

Sub case 2:Let $v_{k} \notin N[v]$.

Then

$$
\sum_{u \in N[v]} g(u)=\underbrace{\frac{1}{q}+\frac{1}{q}+\ldots \ldots+\frac{1}{q}}_{(m+2) \text {-times }}=\frac{m+2}{q}=\frac{m+2}{4}>1 .
$$

Case (iii): Let $v \in C_{m}$ be such that $d(v)=3$ in $G$.

Sub case 1:Let $v_{k} \in N[v]$.

Then $\sum_{u \in N[v]} g(u)=r+\frac{1}{q}+\frac{1}{q}+\frac{1}{q}$

$<\frac{1}{q}+\frac{3}{q}=\frac{4}{4}=1$, since $\mathrm{q}=4$.

Sub case 2:Let $v_{k} \notin N[v]$.

Then $\sum_{u \in N[v]} g(u)=\frac{1}{q}+\frac{1}{q}+\frac{1}{q}+\frac{1}{q}=\frac{4}{4}=1$.

This implies that $\sum_{u \in N[v]} g(u)<1$, for some $\mathrm{v} \in \mathrm{V}$.

So $g$ is not a DF.

Since $g$ is defined arbitrarily, it follows that there exists no $g<f$ such that $g$ is a DF.

Thus $f$ is a MDF. 
Theorem 3.4: A function $f: V \rightarrow[0,1]$ defined by $f(v)=\frac{p}{q}, \forall v \in V \quad$ where $p=\min (m, n)$ and $q=$ $\max (m, n)$ is a DF of $G=P_{n} \odot C_{m}$ if $\frac{p}{q} \geq \frac{1}{4}$. Otherwise it is not a DF. Also it becomes a MDF if $\frac{p}{q}=\frac{1}{4}$.

Proof:Let $f: V \rightarrow[0,1]$ be defined by $f(v)=\frac{p}{q}, \forall v \in V$, where $p=\min (m, n)$ and $q=\max (m, n)$.

Clearly $\frac{p}{q}>0$.

The summation value taken over $N[v]$ of $v \in V$ is as follows:

Case 1: Let $v \in P_{n}$ be such that $d(v)=m+2$ in $G$.

Then $N[v]$ contains $m$ vertices of $C_{m}$ and three vertices of $P_{n}$ in $G$.

So $\sum_{u \in N[v]} f(u)=\underbrace{\frac{p}{q}+\frac{p}{q}+\ldots \ldots+\frac{p}{q}}_{(m+3) \text {-times }}=(m+3) \frac{p}{q}$.

Case 2: Let $v \in P_{n}$ be such that $d(v)=m+1$ in $G$.

Then $N[v]$ contains $m$ vertices of $C_{m}$ and two vertices of $P_{n}$ in $G$.

So $\sum_{u \in N[v]} f(u)=\underbrace{\frac{p}{q}+\frac{p}{q}+\ldots \ldots+\frac{p}{q}}_{(m+2) \text {-times }}=(m+2) \frac{p}{q}$.

Case 3: Let $v \in C_{m}$ be such that $d(v)=3$ in $G$.

Then $N[v]$ contains 3 vertices of $C_{m}$ and one vertex of $P_{n}$ in G.

So $\sum_{u \in N[v]} f(u)=\frac{p}{q}+\frac{p}{q}+\frac{p}{q}+\frac{p}{q}=4\left(\frac{p}{q}\right)$.

From the above three cases, we observe that $f$ is a DF if $\frac{p}{q} \geq \frac{1}{4}$.

Otherwise $f$ is not a DF.

Case 4: Suppose $\frac{p}{q}>\frac{1}{4}$.

Clearly $f$ is a DF.

Now we check for the minimality of $f$.

Define $g: V \rightarrow[0,1]$ by

$$
\begin{aligned}
& g(v) \\
& = \begin{cases}\mathrm{r}, & \text { if } \mathrm{v}=\mathrm{v}_{\mathrm{k}} \in \mathrm{D} \text { with } \mathrm{d}\left(\mathrm{v}_{\mathrm{k}}\right)=\mathrm{m}+1, \\
\frac{\mathrm{q}}{\mathrm{q}}, & \text { otherwise. }\end{cases}
\end{aligned}
$$

where $0<r<\frac{p}{q}$.

Since strict inequality holds at a vertex $v_{k}$ of $V$, it follows that $g<f$.

Case (i): Let $v \in P_{n}$ be such that $d(v)=m+2$ in $G$.
Sub case 1:Let $v_{k} \in N[v]$.

Then $\sum_{u \in N[v]} g(u)=r+\underbrace{\frac{p}{q}+\frac{p}{q}+\ldots \ldots+\frac{p}{q}}_{(m+2) \text {-times }}$

$$
<\frac{p}{q}+(m+2) \frac{p}{q}=(m+3) \frac{p}{q}>1,
$$

since $\frac{p}{q}>\frac{1}{4}$.

Sub case 2: Let $v_{k} \notin N[v]$.

Then

$$
\sum_{u \in N[v]} g(u)=\underbrace{\frac{p}{q}+\frac{p}{q}+\ldots \ldots . .+\frac{p}{q}}_{(m+3) \text {-times }}=(m+3) \frac{p}{q}>1 .
$$

Case (ii): Let $v \in P_{n}$ be such that $d(v)=m+1$ in $G$.

Sub case 1:Let $v_{k} \in N[v]$.

Then $\sum_{u \in N[v]} g(u)=r+\underbrace{\frac{p}{q}+\frac{p}{q}+\ldots \ldots+\frac{p}{q}}_{(m+1) \text {-times }}$

$$
<\frac{p}{q}+(m+1) \frac{p}{q}=(m+2) \frac{p}{q}>1,
$$

since $\frac{p}{q}>\frac{1}{4}$.

Sub case 2: Let $v_{k} \notin N[v]$.

Then

$$
\sum_{u \in N[v]} g(u)=\underbrace{\frac{p}{q}+\frac{p}{q}+\ldots \ldots . .+\frac{p}{q}}_{(m+2) \text {-times }}=(m+2) \frac{p}{q}>1 .
$$

Case (iii): Let $v \in C_{m}$ be such that $d(v)=3$ in $G$.

Sub case 1:Let $v_{k} \in N[v]$.

Then $\sum_{u \in N[v]} g(u)=r+\frac{p}{q}+\frac{p}{q}+\frac{p}{q}$ $<\frac{p}{q}+3\left(\frac{p}{q}\right)=4\left(\frac{p}{q}\right)>1$, since $\frac{p}{q}>\frac{1}{4}$.

Sub case 2: Let $v_{k} \notin N[v]$.

Then $\sum_{u \in N[v]} g(u)=\frac{p}{q}+\frac{p}{q}+\frac{p}{q}+\frac{p}{q}=4\left(\frac{p}{q}\right)>1$. 
Hence, it follows that $\sum_{u \in N[v]} g(u)>1, \quad \forall \mathrm{v} \in \mathrm{V}$.

Thus $g$ is a DF.

This implies that $f$ is not a MDF.

Case 5: Suppose $\frac{p}{q}=\frac{1}{4}$.

As in Case 1 and 2, we have that

$$
\begin{aligned}
\sum_{u \in N[v]} f(u) & =\underbrace{\frac{p}{q}+\frac{p}{q}+\ldots \ldots .+\frac{p}{q}}_{(m+3)-\text { times }}=(m+3) \frac{p}{q}=(m+3) \frac{1}{4}>1, \\
\text { if } v & \in P_{n} .
\end{aligned}
$$

and

$$
\sum_{u \in N[v]} f(u)=\underbrace{\frac{p}{q}+\frac{p}{q}+\ldots \ldots . .+\frac{p}{q}}_{(m+2) \text {-times }}=(m+2) \frac{p}{q}=(m+2) \frac{1}{4}>1,
$$

if $v \in P_{n}$.

Again as in Case 3, we have if $v \in C_{m}$ then

$$
\sum_{u \in N[v]} f(u)=\frac{p}{q}+\frac{p}{q}+\frac{p}{q}+\frac{p}{q}=4\left(\frac{p}{q}\right)=1
$$

Therefore for all possibilities, we get $\sum_{u \in N[v]} f(u) \geq 1$,

$$
\forall \mathrm{v} \in \mathrm{V} .
$$

This implies that $f$ is a DF.

Now we check for the minimality of $f$.

Define $g: V \rightarrow[0,1]$ by

$$
\begin{aligned}
& g(v) \\
& = \begin{cases}\mathrm{r}, & \text { if } \mathrm{v}=\mathrm{v}_{\mathrm{k}} \in \mathrm{D} \text { with } \mathrm{d}\left(\mathrm{v}_{\mathrm{k}}\right)=\mathrm{m}+1, \\
\frac{\mathrm{p}}{\mathrm{q}}, & \text { otherwise. }\end{cases}
\end{aligned}
$$

where $0<r<\frac{p}{q}$.

Since strict inequality holds at a vertex $v_{k}$ of $V$, it follows that $g<f$.

Then we can show as in Case (i) of Case 4 that

$$
\sum_{u \in N[v]} g(u)=r+\underbrace{\frac{p}{q}+\frac{p}{q}+\ldots \ldots+\frac{p}{q}}_{(m+2) \text {-times }}>1,
$$

if $\mathrm{v} \in \mathrm{P}_{\mathrm{n}}$ and $\mathrm{v}_{\mathrm{k}} \in \mathrm{N}[\mathrm{v}]$.

And

$$
\sum_{u \in N[v]} g(u)=\underbrace{\frac{p}{q}+\frac{p}{q}+\ldots \ldots . .+\frac{p}{q}}_{(m+3) \text {-times }}>1,
$$

if $\mathrm{v} \in \mathrm{P}_{\mathrm{n}}$ and $v_{k} \notin N[v]$.

Again as in Case (ii) of Case 4, we can show that

$$
\sum_{u \in N[v]} g(u)=r+\underbrace{\frac{p}{q}+\frac{p}{q}+\ldots \ldots+\frac{p}{q}}_{(m+1) \text {-times }}>1,
$$

if $\mathrm{v} \in \mathrm{P}_{\mathrm{n}}$ and $\mathrm{v}_{\mathrm{k}} \in \mathrm{N}[\mathrm{v}]$.

And

$$
\sum_{u \in N[v]} g(u)=\underbrace{\frac{p}{q}+\frac{p}{q}+\ldots \ldots . .+\frac{p}{q}}=(m+2) \frac{p}{q}=(m+2) \frac{p}{q}>1,
$$

if $\mathrm{v} \in \mathrm{P}_{\mathrm{n}}$ and $v_{k} \notin N[v]$.

Again as in Case (iii) of Case 4, we can show that

$\sum_{u \in N[v]} g(u)=r+\frac{p}{q}+\frac{p}{q}+\frac{p}{q} \quad$ if $\quad \mathrm{v} \in \mathrm{C}_{\mathrm{m}}$ and $\mathrm{v}_{\mathrm{k}} \in$ $\mathrm{N}[\mathrm{v}]$.

$<\frac{p}{q}+3\left(\frac{p}{q}\right)=4\left(\frac{p}{q}\right)=1, \quad$ since $\frac{p}{q}=\frac{1}{4}$.

And $\quad \sum_{u \in N[v]} g(u)=\frac{p}{q}+\frac{p}{q}+\frac{p}{q}+\frac{p}{q}=4\left(\frac{p}{q}\right)=1$, if $\mathrm{v} \in \mathrm{C}_{\mathrm{m}}$ and $v_{k} \notin N[v]$.

This implies that $\sum_{u \in N[v]} g(u)<1$, for some $\mathrm{v} \in \mathrm{V}$.

So $g$ is not a DF.

Since $g$ is defined arbitrarily, it follows that there exists no $g<f$ such that $g$ is a DF.

Thus $f$ is a MDF. 


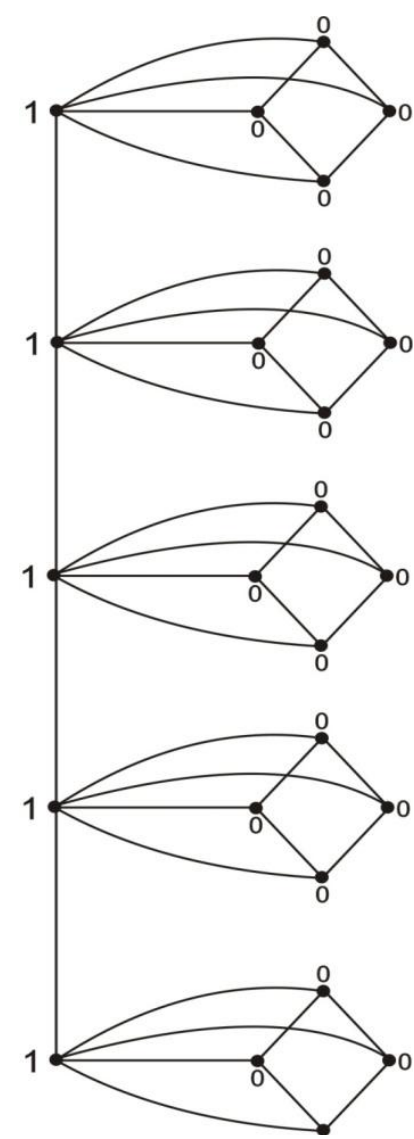

Fig.1

$G=P_{5} \odot C_{4}$

Fig.1: The function $\mathrm{f}$ takes the value 1 for vertices of $P_{n}$ and value 0 for vertices in each copy of $C_{m}$

\section{CONCLUSION}

It is interesting to study the minimal dominating functions of the corona product graph of a path with a cycle. This work gives the scope for the study of convexity of these minimal dominating functions and the authors have also studied this concept.

\section{REFERENCES}

[1] Haynes, T.W, Hedetniemi, S.T and Slater, P.J Domination in Graphs: Advanced Topics, Marcel Dekker, Ic, New York, (1998).

[2] Haynes, T.W, Hedetniemi, S.T and Slater, P.J Fundamentals of domination in graphs, Marcel Dekker, Inc. New York, (1998).

[3] Allan, R.B and Laskar, R.C - On domination, independent domination numbers of a graph, Discrete Math., 23(1978), 73-76.

[4] Cockayne, E.J and Hedetniemi, S.T - Towards a theory of domination in graphs, Networks, 7(1977), $247-261$.

[5] Cockayne, E.J, Mynhardt, C.M and Yu.B-Total dominating functions in trees: Minimality and convexity, Journal of Graph Theory, 19(1995), 83-92.

[6] Jeelani Begum.S - Some studies on dominating functions of Quadratic Residue Cayley Graphs, Ph.D Thesis, Sri Padmavathi Mahila Viswa Vidyalayam, Tirupati, Andhra Pradesh, India(2001).

[7] Frutch, R. and Harary.F - On the corona of two graphs, Awquationes Mathematicae, Volume 4, Issue 3 (1970), 322-325.

[8] Siva Parvathi.M - Some studies on dominating functions of corona product graphs, Ph.D Thesis, Sri Padmavathi Mahila Viswa Vidyalayam, Tirupati, Andhra Pradesh, India(2013).

[9] Berge.C, The Theory of Graphs and its Applications, Methuen, London (1962). 